PROCEEDINGS OF THE

AMERICAN MATHEMATICAL SOCIETY

Volume 136, Number 6, June 2008, Pages 1997-2006

S 0002-9939(08)09032-1

Article electronically published on February 19, 2008

\title{
SEMI-COMPACTNESS OF POSITIVE DUNFORD-PETTIS OPERATORS ON BANACH LATTICES
}

\author{
BELMESNAOUI AQZZOUZ, REDOUANE NOUIRA, AND LARBI ZRAOULA
}

(Communicated by Joseph A. Ball)

\begin{abstract}
We investigate Banach lattices on which each positive DunfordPettis operator is semi-compact and the converse. As an interesting consequence, we obtain Theorem 2.7 of Aliprantis-Burkinshaw and an element of Theorem 1 of Wickstead.
\end{abstract}

\section{INTRODUCTION AND NOTATION}

An operator $T$ from a Banach space $E$ into a Banach lattice $F$ is said to be semicompact if for each $\varepsilon>0$, there exists some $u \in F^{+}$such that $T\left(B_{E}\right) \subset[-u, u]+$ $\varepsilon B_{F}$ where $B_{H}$ is the closed unit ball of $H=E$ or $F$ and $F^{+}=\{x \in F: 0 \leq x\}$. An operator $T$ between two Banach spaces $E$ and $F$ is said to be Dunford-Pettis if the image of each weakly compact subset of $E$ is a compact subset of $F$.

In contrast to compact operators, the class of semi-compact (resp. DunfordPettis) operators does not satisfy the analogue of Schauder's Theorem. However, the class of semi-compact operators satisfies the domination problem (Theorem 18.20 of [3]), but the class of Dunford-Pettis operators fails to satisfy this property, as was proved in [1, 7] and [9].

Finally, a semi-compact operator is not necessarily Dunford-Pettis and, conversely, a Dunford-Pettis operator is not necessarily semi-compact.

In [4] and [5] we studied the compactness of the class of positive Dunford-Pettis operators. And in 6] we characterized Banach lattices on which each positive Dunford-Pettis operator is weakly compact. Also, it follows from Aliprantis and Burkinshaw (1], Theorem 3.4) that if $E$ and $F$ are two Banach lattices such that $E$ has an order-continuous norm, then each regular Dunford-Pettis operator is AMcompact. Our objective in this paper is to continue the invistigation of Banach lattices on which each positive Dunford-Pettis operator is semi-compact and the converse. We will characterize Banach lattices for which each positive DunfordPettis operator is semi-compact, and we will give some interesting consequences. Next, we will prove a sufficient condition under which a positive semi-compact operator is weakly compact. More precisely, we will show that if the norm of a Banach lattice $F$ is order continuous, then each positive semi-compact operator

Received by the editors February 24, 2006 and, in revised form, December 2, 2006.

2000 Mathematics Subject Classification. Primary 46A40, 46B40, 46B42.

Key words and phrases. Semi-compact operator, Dunford-Pettis operator, order continuous norm, discrete vector lattice. 
from a Banach lattice $E$ into $F$ is weakly compact. As consequence, we shall obtain some conditions for which the class of Dunford-Pettis operators, the class of semicompact operators, the class of weakly compact operators, and the class of compact operators coincide. Finally, whenever $E$ is an order $\sigma$-complete Banach lattice, we will establish that if each positive semi-compact operator from $E$ into $E$ is weakly compact (resp. Dunford-Pettis), then the norm of $E$ is order continuous.

To state our results, we need to fix some notation and recall some definitions. A vector lattice $E$ is an ordered vector space in which $\sup (x, y)$ exists for every $x, y$ $\in E$. A subspace $F$ of a vector lattice $E$ is said to be a sublattice if for every pair of elements $a, b$ of $F$ the supremum of $a$ and $b$, taken in $E$, belongs to $F$. A subset $B$ of a vector lattice $E$ is said to be solid if it follows from $|y| \leq|x|$ with $x \in B$ and $y \in E$ that $y \in B$. An order ideal of $E$ is a solid subspace. Let $E$ be a vector lattice, for each $x, y \in E$ with $x \leq y$, the set $[x, y]=\{z \in E: x \leq z \leq y\}$ is called an order interval. A subset of $E$ is said to be order bounded if it is included in some order interval. A Banach lattice is a Banach space $(E,\|\|$.$) such that E$ is a vector lattice and its norm satisfies the following property: for each $x, y \in E$ such that $|x| \leq|y|$, we have $\|x\| \leq\|y\|$. If $E$ is a Banach lattice, its topological dual $E^{\prime}$, endowed with the dual norm, is also a Banach lattice. For more detail about Banach lattices, the reader is referred to the book of Zaanen [10].

\section{MAJOR RESUlts}

We will use the term operator $T: E \longrightarrow F$ between two Banach lattices to mean a bounded linear mapping. It is positive if $T(x) \geq 0$ in $F$ whenever $x \geq 0$ in $E$. An operator $T: E \longrightarrow F$ is regular if $T=T_{1}-T_{2}$ where $T_{1}$ and $T_{2}$ are positive operators from $E$ into $F$. It is well known that each positive linear mapping on a Banach lattice is continuous.

Also, a norm $\|$.$\| of a Banach lattice E$ is order continuous if for each net $\left(x_{\alpha}\right)$ such that $x_{\alpha} \downarrow 0$ in $E$, the sequence $\left(x_{\alpha}\right)$ converges to 0 for the norm $\|\cdot\|$ where the notation $x_{\alpha} \downarrow 0$ means that $\left(x_{\alpha}\right)$ is decreasing, its infimum exists, and $\inf \left(x_{\alpha}\right)=0$. For example, the norm of the Banach lattice $l^{1}$ is order continuous but the norm of the Banach lattice $l^{\infty}$ is not.

To prove the next theorem, we need the following lemma:

Lemma 2.1. Let $E, F$ be Banach lattices and let $T$ be a positive Dunford-Pettis operator from $E$ into $F$. If the topological dual $E^{\prime}$ of $E$ has an order continuous norm, then for each $\varepsilon>0$, there exists some $y \in E^{+}$such that

$$
T\left(B_{E} \cap E^{+}\right) \subset \varepsilon B_{F}+T([0, y]),
$$

where $B_{H}$ is the closed unit ball of $H=E, F$.

Proof. It follows from the proof of Theorem 2.7 and Theorem 2.8 of [1].

Recall that a nonzero element $x$ of a vector lattice $E$ is discrete if the order ideal generated by $x$ equals the subspace generated by $x$. The vector lattice $E$ is discrete if it admits a complete disjoint system of discrete elements. For example, the Banach lattice $l^{1}$ is discrete but $C([0,1])$ is not discrete.

A Dunford-Pettis operator is not necessarily semi-compact. In fact, the identity operator $\operatorname{Id}_{c}: c \longrightarrow c$ is semi-compact but it is not Dunford-Pettis where $c$ is the Banach lattice of all convergent sequences. The following theorem gives a 
sufficient and necessary condition for which a regular Dunford-Pettis operator is semi-compact. In fact,

Theorem 2.2. Let $E$ be a Banach lattice. Then the following assertions are equivalent:

1. E' has an order continuous norm.

2. Each positive Dunford-Pettis operator from $E$ into $F$ is semi-compact for each Banach lattice $F$.

3. Each positive Dunford-Pettis operator from $E$ into $E$ is semi-compact.

Proof. For the implication $1 \Longrightarrow 2$. Let $F$ be a Banach lattice and let $T$ be a positive Dunford-Pettis operator from $E$ into $F$. It follows from Lemma 2.1 that for each $\varepsilon>0$, there exists some $y \in E^{+}$such that

$$
T\left(B_{E}^{+}\right) \subset \varepsilon B_{F}^{+}+T([0, y]),
$$

where $B_{H}^{+}=B_{H} \cap E^{+}$for $H=E, F$.

Since

$$
T([0, y]) \subset[0, T(y)],
$$

we obtain

$$
T\left(B_{E}^{+}\right) \subset \varepsilon B_{F}^{+}+[0, T(y)] .
$$

This proves the result.

For the implication $3 \Longrightarrow 1$. If the norm of $E^{\prime}$ is not order continuous, then it follows from the proof of Theorem 1 of Wickstead [9], that $E$ contains a sublattice that is isomorphic to $l^{1}$ and there exists a positive projection $P$ from $E$ into $l^{1}$.

Consider the operator product

$$
i \circ P: E \longrightarrow l^{1} \longrightarrow E,
$$

where $i$ is the inclusion operator of $l^{1}$ in $E$. It is clear that $i \circ P$ is a positive Dunford-Pettis operator. We have to prove that $i \circ P$ is not semi-compact. If not, its restriction to $l^{1}$ is a semi-compact operator, and then the restriction of the operator $P \circ(i \circ P)$ to $l^{1}$, which coincides with the operator identity $\operatorname{Id}_{l^{1}}$ of $l^{1}$, is semi-compact; i.e. for each $\varepsilon>0$, there exists some $y \in\left(l^{1}\right)^{+}$such that

$$
B_{l^{1}} \subset \varepsilon B_{l^{1}}+[-y, y] .
$$

Since the Banach lattice $l^{1}$ is discrete and has an order continuous norm, it follows from Theorem 3.22 of 2 that the order interval $[-y, y]$ is compact in $l^{1}$. Hence, the closed unit ball $B_{l^{1}}$ is precompact. This presents a contradiction.

The implication $2 \Longrightarrow 3$ is trivial.

Remark 2.3. If we fix the Banach lattice $F$, the implication $2 \Longrightarrow 1$ of Theorem 2.2. is false. In fact, if we take $F$ of finite dimension and $E=l^{1}$, it is clear that each positive Dunford-Pettis operator from $l^{1}$ into $F$ is semi-compact, but it is well known that the norm of the topological dual $E^{\prime}=l^{\infty}$ is not order continuous.

Recall from Zaanen [10] that a regular operator $T$ from a vector lattice $E$ into a Banach lattice $F$ is said to be AM-compact if it carries each order-bounded subset of $E$ onto a relatively compact subset of $F$.

As a consequence of Theorem 2.2 of [6] and the above theorem, we obtain 
Corollary 2.4. Let $E$ be a Banach lattice. Then the following statements are equivalent:

1. Each positive Dunford-Pettis operator from $E$ into $E$ is weakly compact.

2. Each positive Dunford-Pettis operator from $E$ into $E$ is semi-compact.

3. For each positive Dunford-Pettis operator $T$ from $E$ into $E$, the second power operator $T^{2}$ is compact.

4. For each pair of operators $S$ and $T$ from $E$ into $E$ such that $0 \leq S \leq T$ and $T$ is Dunford-Pettis, the operator $S$ is weakly compact.

5. The norm of the topological dual $E^{\prime}$ is order continuous.

The following result is a consequence of a theorem of Dodds and Fremlin ([10], Theorem 125.5) and Theorem 2.2:

Corollary 2.5. Let $E$ and $F$ be two Banach lattices such that $E^{\prime}$ and $F$ have order continuous norms. Let $T$ be a positive operator from $E$ into $F$. Then the following assertions are equivalent:

1. $T$ is compact.

2. $T$ is Dunford-Pettis and AM-compact.

3. $T$ is semi-compact and AM-compact.

Now by combining our Corollary 2.5 and Theorem 3.5 of Aliprantis and Burkinshaw [1], we obtain Theorem 2.7 of [1] as a consequence, which was the basic result of our papers [4] and [5].

Corollary 2.6. Let $E$ be a Banach lattice such that $E$ and its topological dual $E^{\prime}$ have order continuous norms. Then each positive Dunford-Pettis operator from $E$ into $E$ is compact.

Proof. Let $T$ be a positive Dunford-Pettis operator from $E$ into $E$. Since the norm of $E$ is order continuous, it follows from Theorem 3.5 of [1] that $T$ is AM-compact. Finally, Corollary 2.5 implies that $T$ is compact.

Let $T$ be a positive operator from a Banach lattice $E$ into a Banach lattice $F$. If $T^{\prime}$ is the adjoint operator from $F^{\prime}$ into $E^{\prime}$ defined by $T^{\prime}(f)(x)=f(T(x))$ for each $f \in F^{\prime}$ and for each $x \in E$, it is clear that $T^{\prime}$ is positive.

Recall that a Banach space $E$ has the Dunford-Pettis property if and only if each weakly compact operator on $E$, taking its value in another Banach space, is Dunford-Pettis.

Now, to give a sufficient condition under which each positive semi-compact operator is Dunford-Pettis, we study the compactness of a positive semi-compact operator.

Theorem 2.7. Let $E$ and $F$ be Banach lattices. Then each positive semi-compact operator from $E$ into $F$ is compact if one of the following statements is valid:

1. $F$ is discrete and its norm is order continuous.

2. $E^{\prime}$ is a discrete order continuous norm, and $F$ has an order continuous norm.

3. The norms of $E, E^{\prime}$ and $F$ are order continuous, and $E$ has the DunfordPettis property.

Proof. 1. Let $T$ be a positive semi-compact operator from $E$ into $F$. By Theorem 3.22 of [1, for each $y \in F^{+}$, the order interval $[0, y]$ is norm compact in $F$. On the other hand, for each $\varepsilon>0$, there exists some $x \in F^{+}$such that

$$
T\left(B_{E}\right) \subset \varepsilon B_{F}+[-x, x] .
$$


This proves that $T\left(B_{E}\right)$ is norm relatively compact in $F$ and hence $T$ is compact.

2. Let $T$ be a positive semi-compact operator from $E$ into $F$. Since the norms of $E^{\prime}$ and $F$ are order continuous, then the adjoint operator $T^{\prime}$ from $F^{\prime}$ into $E^{\prime}$ is semi-compact (Theorem 125.6 of [10]). As the Banach lattice $E^{\prime}$ is discrete and its norm is order continuous, then condition 1 implies that $T^{\prime}: F^{\prime} \longrightarrow E^{\prime}$ is compact, and therefore $T$ is compact.

3. Let $T$ be a positive semi-compact operator from $E$ into $F$. Since $F$ has an order continuous norm, it follows from Theorem 2.2 that $T$ is weakly compact. Now, the Dunford-Pettis property of $E$ implies that $T$ is Dunford-Pettis. On the other hand, $E$ and $E^{\prime}$ have order continuous norms, so Theorem 2.7 of Aliprantis and Burkinshaw [1] implies that $T$ is compact.

As an immediate consequence of Theorem 2.6, we obtain a result of Wickstead ([8], Proposition 2.3 or [9], Theorem 1):

Corollary 2.8. Let $E$ and $F$ be Banach lattices, and let $S$ and $T$ be positive operators from $E$ into $F$ such that $0 \leq S \leq T$ and $T$ is compact. If $F$ is discrete and its norm is order continuous, then $S$ is compact.

Recall that a semi-compact operator is not necessarily Dunford-Pettis. For example, the identity operator $\operatorname{Id}_{l^{1}}: l^{1} \longrightarrow l^{1}$ is Dunford-Pettis but it is not semicompact. Another consequence of Theorem 2.7 is the following:

Corollary 2.9. Let $E$ and $F$ be Banach lattices. If $F$ is discrete and its norm is order continuous, then each positive semi-compact operator from $E$ into $F$ is Dunford-Pettis.

Recall that a semi-compact operator is not necessarily weakly compact, and conversely a weakly compact operator is not necessarily semi-compact. For example, the identity operator $\operatorname{Id}_{l^{2}}: l^{2} \longrightarrow l^{2}$ is weakly compact but it is not semi-compact (if not, since $l^{2}$ is discrete and its norm is order continuous, it follows from Theorem 2.7 (1) that $\operatorname{Id}_{l^{2}}$ is compact) and conversely, the identity operator $\operatorname{Id}_{c}: c \longrightarrow c$ is semi-compact but it is not weakly compact.

The following proposition gives a sufficient condition under which a positive weakly compact operator is semi-compact:

Proposition 2.10. Let $E$ and $F$ be two Banach lattices. If $E$ has the DunfordPettis property and its topological dual $E^{\prime}$ has an order continuous norm, then each positive weakly compact operator from $E$ into $F$ is semi-compact.

Proof. Let $T$ be a positive weakly compact operator from $E$ into $F$. Since $E$ admits the Dunford-Pettis property, then $T$ is Dunford-Pettis. Now the semi-compactness of $T$ follows from the order continuousness of the norm of the topological dual $E^{\prime}$ (Theorem 2.2).

Conversely, we give a sufficient condition under which a positive semi-compact operator is weakly compact.

Theorem 2.11. Let $E$ and $F$ be two Banach lattices. If the norm of $F$ is order continuous, then each positive semi-compact operator from $E$ into $F$ is weakly compact. 
Proof. Let $T$ be a positive semi-compact operator from $E$ into $F$. Then for each $\varepsilon>$ 0 , there exists some $y \in F^{+}$such that

$$
T\left(B_{E} \cap E^{+}\right) \subset \varepsilon B_{F}+[0, y],
$$

where $B_{H}$ is the unit ball of $H=E, F$. Since $F$ has an order continuous norm, then the order interval $[0, y]$ is weakly compact, and hence $T\left(B_{E} \cap E^{+}\right)$is weakly precompact. To prove that $T\left(B_{E} \cap E^{+}\right)$is weakly relatively compact, it is sufficient to show that the closure of $T\left(B_{E} \cap E^{+}\right)$, for the topology $\sigma\left(F, F^{\prime}\right)$, is weakly complete. To show this, we use the same proof as Theorem 2.2 of [6]. In fact, let $\left(T\left(x_{i}\right)\right)_{i}$ be a Cauchy net for the topology $\sigma\left(F, F^{\prime}\right)$, where $\left(x_{i}\right)$ is a net in $B_{E} \cap E^{+}$. Since $T\left(B_{E} \cap E^{+}\right)$is relatively compact for $\sigma\left(F^{\prime \prime}, F^{\prime}\right)$ in the topological bidual $F^{\prime \prime}$, the sequence $\left(T\left(x_{i}\right)\right)_{i}$ converges to some $\Psi \in F^{\prime \prime}$ for $\sigma\left(F^{\prime \prime}, F^{\prime}\right)$. Let $m \in \mathbb{N}^{*}$; it follows from (1), the existence of $y^{m} \in F^{+}, z_{i}^{m} \in B_{F}$ and $w_{i}^{m} \in\left[0, y^{m}\right]$ such that

$$
T\left(x_{i}\right)=\frac{1}{m} z_{i}^{m}+w_{i}^{m} .
$$

On the other hand, since $\left[0, y^{m}\right]$ is relatively weakly compact, there exists an accumulation point $a_{m} \in F$ of the net $\left(w_{i}^{m}\right)_{i}$.

If we fix $f \in F^{\prime}$ with $\|f\| \leq 1$, then there exists some $i_{0} \in I$ such that for each $i>i_{0}$, we have

and hence

$$
\left|f \circ T\left(x_{i}\right)-\Psi(f)\right|<\frac{1}{m},
$$

$$
\left|f\left(w_{i}^{m}\right)-\Psi(f)\right|<\frac{2}{m} .
$$

Since $a_{m}$ is an accumulation point of the sequence $\left(w_{i}^{m}\right)_{i}$, there exists some $i>i_{0}$ such that

This implies that

$$
\left|f\left(w_{i}^{m}\right)-f\left(a_{m}\right)\right|<\frac{1}{m} .
$$

$$
\left|\Psi(f)-f\left(a_{m}\right)\right|<\frac{3}{m}
$$

and so

$$
\left\|\Psi-a_{m}\right\|_{F^{\prime \prime}} \leq \frac{3}{m}
$$

where $\|\cdot\|_{F^{\prime \prime}}$ is the norm of $F^{\prime \prime}$. It follows that $\left(a_{m}\right)$ converges in norm to $\Psi$, and hence $\Psi \in F$. This proves that the closure of $T\left(B_{E} \cap E^{+}\right)$for $\sigma\left(F, F^{\prime}\right)$ in $F$ coincides with its closure in $\hat{F}$ where $\hat{F}$ is the completion of $F$ for $\sigma\left(F, F^{\prime}\right)$. Hence, ${\overline{T\left(B_{E} \cap E^{+}\right)}}^{\sigma\left(F, F^{\prime}\right)}$ is complete.

A Banach lattice $E$ is said to be an AM-space if for each $x, y \in E$ such that $\inf (x, y)=0$, we have $\|x+y\|=\max \{\|x\|,\|y\|\}$. It is an AL-space if its topological dual $E^{\prime}$ is an AM-space. For example, the Banach lattice $l^{1}$ is an AL-space and the Banach lattice $l^{\infty}$ is an AM-space.

As consequence of Theorem 2.11, we have:

Corollary 2.12. Let $E$ be an AM-space with unit and $F$ be a Banach lattice with an order continuous norm. Then each positive operator from $E$ into $F$ is weakly compact. In particular, each positive operator from $E$ into $l^{1}$ is compact. 
Proof. Let $T: E \longrightarrow F$ be a positive operator. As $E$ is an AM-space with unit, it follows that $T$ is semi-compact. Now, since $F$ has an order continuous norm, Theorem 2.11 implies that $T$ is weakly compact.

Finally, let $S: E \longrightarrow l^{1}$ be a positive operator where $E$ is an AM-space with unit. Since $l^{1}$ has an order continuous norm, the operator $S$ is weakly compact. On the other hand, $S=\operatorname{Id}_{l^{1}} \circ S$, where $\operatorname{Id}_{l^{1}}: l^{1} \longrightarrow l^{1}$ is the identity operator of $l^{1}$ which is Dunford-Pettis; hence, $S$ is compact.

Remark 2.13. If $E$ is a Banach lattice with a discrete topological dual $E^{\prime}$, then there exists a positive semi-compact operator which is not weakly compact. In fact, if we take $E=F=c$, then $E^{\prime}$ is discrete, and the identity operator $\operatorname{Id}_{E}$ of $E$ is semi-compact but it is not weakly compact.

The following consequence of Theorem 2.11 gives another sufficient condition under which each positive semi-compact operator is Dunford-Pettis.

Corollary 2.14. Let $E$ and $F$ be two Banach lattices. If $E$ has the Dunford-Pettis property and the norm of $F$ is order continuous, then each positive semi-compact operator is Dunford-Pettis.

Proof. Let $T$ be a positive semi-compact operator from $E$ into $F$. Since the norm of $F$ is order continuous, it follows from Theorem 2.11 that $T$ is weakly compact. As $E$ has the Dunford-Pettis property, the operator $T$ is Dunford-Pettis.

Another consequence of Theorem 2.11 is the following:

Corollary 2.15. Let $E$ and $F$ be two Banach lattices such that $E^{\prime}$ and $F$ have order continuous norms and $E$ has the Dunford-Pettis property. Let $T: E \longrightarrow F$ be a positive operator. Then the following assertions are equivalent:

1. $T$ is Dunford-Pettis.

2. $T$ is semi-compact.

3. $T$ is weakly compact.

In particular, if we take $E=F$, under the same conditions as Corollary 2.15, we have the following result:

Corollary 2.16. Let $E$ be a Banach lattice having the Dunford-Pettis property such that $E$ and $E^{\prime}$ have order continuous norms. Let $T: E \longrightarrow E$ be a positive operator. Then the following assertions are equivalent:

1. $T$ is Dunford-Pettis.

2. $T$ is semi-compact.

3. $T$ is weakly compact.

4. $T$ is compact.

Remark 2.17. Let $E$ be a Banach lattice having the Dunford-Pettis property. If the topological dual $E^{\prime}$ is discrete with an order continuous norm, there exists a positive semi-compact operator which is not Dunford-Pettis. In fact, the identity operator $\operatorname{Id}_{c}$ of $c$ is a positive semi-compact operator which is not Dunford-Pettis. However, the topological dual $c^{\prime}$ is discrete, $c$ has the Dunford-Pettis property, and $c^{\prime}$ has an order continuous norm.

Whenever $E=F$ and $E$ is order $\sigma$-complete (i.e. every majorized countable nonempty subset of $E$ has a supremum), we obtain the following converse of Theorem 2.11: 
Theorem 2.18. Let $E$ be an order $\sigma$-complete Banach lattice. If each positive semi-compact operator from $E$ into $E$ is weakly compact (resp. Dunford-Pettis), then the norm of $E$ is order continuous.

Proof. Assume that the norm of $E$ is not order continuous. Since $E$ is order $\sigma$ complete, it follows from the proof of Theorem 1 of Wickstead [9] that $E$ contains a sublattice which is isomorphic to $l^{\infty}$ and there exists a positive projection $P$ : $E \longrightarrow l^{\infty}$.

If we take the operator

$$
i \circ P: E \longrightarrow l^{\infty} \longrightarrow E,
$$

it is clear that $i \circ P$ is a semi-compact operator which is not weakly compact (resp. Dunford-Pettis). If not, the restriction of the operator $P \circ(i \circ P)$ to $l^{\infty}$, which coincides with the identity operator $\operatorname{Id}_{l}$ of $l^{\infty}$, would be weakly compact (resp. Dunford-Pettis). This presents a contradiction.

Recall that Wickstead characterized Banach lattices which satisfy the problem of domination for the class of positive weakly compact operators ([8], Theorem 17.10). We observe that by the same proof as Theorem 17.10 of Wickstead [8, we obtain the following result:

Theorem 2.19. Let $E$ and $F$ be two Banach lattices. If every positive operator from $E$ into $F$ dominated by a compact operator is weakly compact, then either $E^{\prime}$ or $F$ has an order continuous norm.

Proof. If the norms of $F$ and $E^{\prime}$ are not order continuous, Wickstead constructed in the proof of 8 , Theorem 17.10 two positive operators $S, T: E \longrightarrow F$ such that $0 \leq S \leq T$ and $T$ is compact but $S$ is not weakly compact. This proves the result.

Whenever $E \neq F$, by combining Theorem 2.19 with Theorem 18.20 of 3 ] we obtain

Theorem 2.20. Let $E$ and $F$ be two Banach lattices. If each positive semi-compact operator from $E$ into $F$ is weakly compact, then one of the following assertions is valid:

1. F has an order continuous norm.

2. $E^{\prime}$ has an order continuous norm.

Proof. In fact, let $S, T: E \longrightarrow F$ be two operators such that $0 \leq S \leq T$ and $T$ is compact. Since $T$ is semi-compact, it follows that $S$ is semi-compact (Theorem 18.20 of [3]). Hence $S$ is weakly compact and the result comes from Theorem 2.19.

Also, recall that Wickstead (9], Theorem 2) studied the converse of the domination problem of the class of positive Dunford-Pettis operators. We remark that by the same proof as Theorem 2 of [9], we can establish the following result:

Theorem 2.21. For two Banach lattices $E$ and $F$, the following assertions are equivalent:

1. Every positive operator from $E$ into $F$ dominated by a compact operator is Dunford-Pettis.

2. The norm of $F$ is order continuous or the lattice operations of $E$ are weakly sequentially continuous. 
Proof. If the norm of $F$ is not order continuous and the lattice operations of $E$ are not weakly sequentially continuous, Wickstead constructed in the proof of Theorem 2 of [9] two positive operators $S, T: E \longrightarrow F$ such that $0 \leq S \leq T$ and $T$ is compact but $S$ is not Dunford-Pettis.

A combination of Theorem 2.21 and Theorem 18.20 of [3] gives

Theorem 2.22. Let $E$ and $F$ be Banach lattices. If each positive semi-compact operator from $E$ into $F$ is Dunford-Pettis, then one of the following assertions is valid:

1. The norm of $F$ is order continuous.

2. The lattice operations of $E$ are weakly sequentially continuous.

Proof. Let $S$ and $T$ be two positive operators from $E$ into $F$ such that $0 \leq S \leq T$ and $T$ is compact. Since $T$ is semi-compact, $S$ is semi-compact ([3], Theorem 18.20) and then Dunford-Pettis. Now, the result follows from Theorem 2.21.

Remark 2.23. Let $E$ and $F$ be two Banach lattices such that one of the following properties is valid:

1. The topological dual $E^{\prime}$ of $E$ is discrete.

2. $F$ is discrete.

3. The norm of $E^{\prime}$ is order continuous.

Then we can always find a positive semi-compact operator from $E$ into $F$ which is not Dunford-Pettis. In fact, the identity operator $\operatorname{Id}_{c}: c \longrightarrow c$ is semi-compact which is not Dunford-Pettis but the above three conditions are satisfied by the Banach lattice $c$.

Remark 2.24. There exist Banach lattices $E$ and $F$ and there exists a positive Dunford-Pettis operator from $E$ into $F$ which is semi-compact but not necessarily weakly compact. In fact, we take the Banach lattice $E=F=l^{1} \oplus l^{\infty}$. Since the norms of $E$ and $E^{\prime}$ are not order continuous, it follows, from Theorem 1 of Wickstead [9], the existence of two operators $S$ and $T$ from $E$ into $E$, such that $0 \leq S \leq T$ and $T$ is compact but $S$ is not compact. On the other hand, the lattice operations of $E$ are weakly sequentially continuous (i.e. the sequence $\left(\left|x_{n}\right|\right)$ converges to 0 for the weak topology $\sigma\left(E, E^{\prime}\right)$ whenever the sequence $\left(x_{n}\right)$ converges to 0 for $\sigma\left(E, E^{\prime}\right)$ ), then an application of Theorem 2 of Wickstead 9 implies that $S$ is Dunford-Pettis. Now, as the norms of $E$ and $E^{\prime}$ are not order continuous, the operator $S$ is not necessarily weakly compact ([8], Theorem 2.2 ).

\section{REFERENCES}

[1] Aliprantis, C.D., and Burkinshaw, O., Dunford-Pettis operators on Banach lattices. Trans. Amer. Math. Soc. vol. 274, 1 (1982) 227-238. MR670929 (84b:47045)

[2] Aliprantis, C.D., and Burkinshaw, O., Locally solid Riesz spaces with applications to economics. Second edition. Mathematical Surveys and Monographs, 105. American Mathematical Society, Providence, RI, 2003. MR2011364 (2005b:46010)

[3] Aliprantis, C.D., and Burkinshaw, O., Positive operators. Springer-Verlag, Berlin and Heidelberg, 2006. (This monograph was reprinted by Springer-Verlag in 2006.) MR2262133

[4] Aqzzouz, B., Nouira R., and Zraoula L., Compacité des opérateurs de Dunford-Pettis positifs sur les treillis de Banach. C. R. Math. Acad. Sci. Paris 340, 1 (2005) 37-42. MR 2112038 (2005m:47081)

[5] Aqzzouz, B., Nouira, R., and Zraoula, L., About positive Dunford-Pettis operators on Banach lattices. J. Math. Anal. Appl. 324, 1 (2006) 49-59. MR2262455 
[6] Aqzzouz, B., Nouira, R., and Zraoula, L., Les opérateurs de Dunford-Pettis positifs qui sont faiblement compacts. Proc. Amer. Math. Soc. 134 (2006) 1161-1165. MR2196052 (2006h:46015)

[7] Kalton, N.J., and Saab, P., Ideal properties of regular operators between Banach lattices. Illinois Journal of Math. 29, 3 (1985) 382-400. MR786728 (87a:47064)

[8] Wickstead, A.W., Extremal structure of cones of operators, Quart. J. Math. Oxford (2) 32 (1981) 239-253. MR615198 (82i:47069)

[9] Wickstead, A.W., Converses for the Dodds-Fremlin and Kalton-Saab theorems, Math. Proc. Camb. Phil. Soc. 120 (1996) 175-179. MR.1373356 (96m:47067)

[10] Zaanen, A.C., Riesz spaces II, North Holland Publishing Company, 1983. MR704021 (86b:46001)

Département d’Economie, Faculté des Sciences Economiques, Juridiques et Sociales, Université Mohammed V-Souissi, B.P. 5295, Sala Eljadida, Morocco

E-mail address: baqzzouz@hotmail.com

Université Ibn Tofail, Faculté des Sciences, Département de Mathématiques, B.P. 133, KÉnitra, Morocco

Université Ibn Tofail, Faculté des Sciences, Département de Mathématiques, B.P. 133, KÉnitra, Morocco 